\title{
Monte Carlo determination of the phase diagram of the double-exchange model
}

\author{
J. L. Alonso ${ }^{1}$, J. A. Capitán ${ }^{2}$, L. A. Fernández² ${ }^{\text {, F. Guinea }}{ }^{3}$, and V. Martín-Mayor ${ }^{4}$ \\ ${ }^{1}$ Departamento de Física Teórica, Facultad de Ciencias, Universidad de Zaragoza, 50009 Zaragoza, Spain. \\ 2 Departamento de Física Teórica, Facultad de CC. Físicas, Universidad Complutense de Madrid, 28040 Madrid, Spain. \\ ${ }^{3}$ Instituto de Ciencia de Materiales (CSIC). Cantoblanco, 28049 Madrid. Spain. \\ 4 Dipartimento di Fisica, Università di Roma "La Sapienza", 00185 Roma and INFN sezione di Roma, Italy.
}

(October 24, 2018)

\begin{abstract}
We study the phase diagram of the double exchange model, with antiferromagnetic interactions, in a cubic lattice both at zero and at finite temperature. There is a rich variety of magnetic phases, combined with regions where phase separation takes place. We identify phases, intrinsic to the cubic lattice, which are stable for realistic values of the interactions and dopings. Some of these phases break chiral symmetry, leading to unusual features.
\end{abstract}

75.10.-b, 75.30.Et

\section{INTRODUCTION.}

The main magnetic properties of doped manganites and other materials $11-3$ are described in terms of double exchange interactions [1,.5]. An extensive body of work has been devoted to the understanding of this model, and the related ferromagnetic Kondo lattice, since the pioneering work of de Gennes [6] (for a recent review, see [7]).

While the model is extremely simple to formulate, as it can be written in terms of two dimensionless parameters only (see next section), the elucidation of its phase diagram is yet to be completed. Initial studies [6] suggested that the phase diagram could be understood in terms of a ferromagnetic and an antiferromagnetic phase with an intermediate phase with a canted arrangement of moments. More recent work showed that in the region of the phase diagram where canting was expected, phase separation is more likely 8 [12]. By now, there are extensive studies of the phase diagram of the model in one dimension 99,11, 13, two dimensions [11, 14], and infinite dimensions 12,16 19]. Even in these relatively simple cases, the model shows a rich phase diagram, which is not completely elucidated.

The situation is far from understood in the more relevant case of three dimensions. There is a general consensus that in the limit of infinite Hund's coupling (see next section) the system has a ferromagnetic phase, although phase separation near the Curie temperature at low dopings has also been found [12 20]. If the Hund's coupling is not infinite, or if there are antiferromagnetic superexchange interactions present, the situation becomes more complicated. There is evidence that an antiferromagnetic coupling can induce a first order phase transition at relatively large dopings 21. Emphasis on the presence of first-order transitions at low temperatures has also been done in Refs. 222,23]. In three dimensions, Berry's phases in the wave function of the electrons may arise [24,25, leading to another source of complexity.

The present work presents a numerical study of the double exchange model in three dimensions, using a method discussed elsewhere [26]. We show that the interplay between antiferromagnetic interactions and a three dimensional structure leads to a rich phase diagram, where, in addition to ferro- and antiferromagnetic phases, other ordered phases with cubic symmetry are possible. The model is discussed in the next section. Then, we present the calculation at zero temperature and the finite temperature results. In the last section, we discuss the main features of our findings, and their experimental implications.

\section{THE MODEL.}

\section{A. The Hamiltonian.}

We study the double exchange model. In its simplest form, electrons in a cubic lattice are coupled to localized spins defined at the same lattice sites. The spin of the electrons is constrained to be parallel to that of the localized spins. This restriction leads to a modulation of the electron hopping between lattice sites, which depends on the relative orientation of the core spins. In addition, one can define a direct coupling between spins at different sites. The Hamiltonian is:

$$
\mathcal{H}=-J_{\mathrm{AF}} \sum_{i j} \boldsymbol{S}_{i} \cdot \boldsymbol{S}_{j}+t \sum_{i j}\left(\left\langle\theta_{i} \phi_{i} \mid \theta_{j} \phi_{j}\right\rangle c_{i}^{\dagger} c_{j}+\text { h.c. }\right) .
$$

where $\left\langle\theta_{i} \phi_{i} \mid \theta_{j} \phi_{j}\right\rangle$ is the overlap between the spinors defined by the polar angle $\theta$, and the azimuthal angle $\phi$ :

$$
\left\langle\theta_{i} \phi_{i} \mid \theta_{j} \phi_{j}\right\rangle=\cos \frac{\theta_{i}}{2} \cos \frac{\theta_{j}}{2}+\sin \frac{\theta_{i}}{2} \sin \frac{\theta_{j}}{2} e^{-i\left(\phi_{i}-\phi_{j}\right)} .
$$

We describe the spins $\boldsymbol{S}$ by classical variables, normalized to one, $|\boldsymbol{S}|=1$. The system depends on two dimensionless parameters only, the filling of the electron band, $x$, and the ratio $J_{\mathrm{AF}} / t$ (notice that our $J_{\mathrm{AF}}$ is opposite in sign to the convention of []]

The double exchange Hamiltonian, Eq. (11), can be derived from the model which describes a lattice of atoms with strong intra-atomic Hund's coupling between electrons in different orbitals. The simplest Hamiltonian 
which includes this effect is that of the ferromagnetic Kondo lattice,

$$
\mathcal{H}_{\mathrm{FK}}=t \sum_{i, j, s}\left(c_{i, s}^{\dagger} c_{j, s}+\text { h.c. }\right)-J_{\mathrm{H}} \sum_{i, s, s^{\prime}} c_{i, s}^{\dagger} c_{i, s^{\prime}} \boldsymbol{\sigma}_{s s^{\prime}} \cdot \boldsymbol{S}_{i},
$$

where $J_{\mathrm{H}}>0$, and the $\sigma$ 's are Pauli matrices. When $J_{\mathrm{H}} \gg t$, the spin sub-band with an antiparallel orientation to the core spin lies at high energy, and can be projected out. To first order in $1 / J_{\mathrm{H}}$, one finds an antiferromagnetic interaction between nearest neighbor core spins, leading to the double exchange Hamiltonian, Eq. (11), with $\left|J_{\mathrm{AF}}\right|=t^{2} /\left(4 J_{\mathrm{H}}\right)$ (note that we have normalized the core spins). This effect adds to an antiferromagnetic superexchange interaction between the $\mathrm{Mn}$ $\mathbf{t}_{\mathbf{g}}$ core spins. Therefore, the most phenomenologically interesting range for $\left|J_{\mathrm{AF}}\right| / t$ is $0.008-0.15$ [21].

\section{B. Other interactions.}

The model discussed above is supposed to be a reasonable starting point to the study of the magnetic properties of doped manganites, $\mathrm{La}_{1-x} A_{x} \mathrm{MnO}_{3}$, where $A$ stands for a divalent cation. The electrons of the conduction band arise from the $\mathrm{Mn} \mathbf{e}_{\mathbf{g}}$ orbitals, while the core spin is built up from three electrons in the $\mathrm{Mn} \mathbf{t}_{\mathbf{g}}$ orbitals. In a cubic lattice, the $\mathbf{e}_{\mathbf{g}}$ orbitals are doubly degenerate. This degeneracy is ignored in Eq. (11). Thus, we cannot study effects associated to orbital ordering [27. These effects are probably important in explaining the different phases with charge ordering observed in these materials. The model studied here can be considered a first approximation to the study of the magnetic properties, in situations where there is no charge ordering and the lattice symmetry is cubic.

The existence of double degeneracy in the $\mathbf{e}_{\mathbf{g}}$ orbitals implies the possibility of lattice Jahn-Teller distortions, which have been considered the cause of the breakdown of cubic symmetry at low dopings. In addition, dynamic Jahn-Teller fluctuations can play a role in some properties of doped compounds with cubic symmetry 28. We will ignore these interactions. Previous studies 21] suggest that the model used here suffices to understand the main features of the magnetic transitions in the manganites, in the doping range $0.1 \leq x \leq 0.4$ where most experiments are done.

The treatment of the core spins as classical variables will overestimate the tendency of the system toward long range order. Previous studies [10] suggest that this effect is small, as results obtained for the physical value $S=3 / 2$ are very close to those found in the limit $S \rightarrow \infty$. In addition, the only transitions at zero temperature found in our work are discontinuous (see next section). Hence, quantum spin fluctuations are bounded, and cannot change qualitatively the results.

\section{RESULTS.}

\section{A. Phase diagram at zero temperature.}

The phase diagram at zero temperature can be analyzed by minimizing the total energy, Eq. (1), as function of the spin configuration, for the different values of $x$ and the ratio $J_{\mathrm{AF}} / t$. However, previous studies in one, two and infinite dimensions 8 14] have shown that the system has a strong tendency toward phase-separation. This enforces us to use the Maxwell construction to correctly find the phase boundaries. The easiest way of performing the Maxwell construction consist in minimizing the grand-canonical energy

$$
\mathcal{H}^{\mathrm{GC}}=\mathcal{H}-\mu \sum_{i} c_{i}^{\dagger} c_{i},
$$

as a function of the spin configuration for the different values of $\mu$ and $J_{\mathrm{AF}}$. In this way, we obtain the band filling, $x$, as a function of the chemical potential $\mu / t$ and of $J_{\mathrm{AF}}$. At the phase boundaries $x$ is a discontinuous (growing) function of $\mu$ : the jump at the discontinuity is the phase-separation compositional range.

For the above mentioned minimization we will limit ourselves to the possible spin textures observed in Monte Carlo calculations at finite temperatures (see next subsection) and to those which seem plausible on physical grounds. Some of the most stable phases analyzed are described in Table I. We have studied only the range $x \leq 0.5$, due to the particle-hole symmetry of the Hamiltonian (1).

We find the ferromagnetic (FM) and antiferromagnetic (G-AFM) phases. The A-AFM configuration is ferromagnetic within planes, and antiferromagnetic between neighboring planes. The C-AFM configuration is ferromagnetic along lines, and antiferromagnetic between neighboring lines. These phases have already been discussed in the literature. The twisted phase interpolates between the A-AFM and the C-AFM phases. Island phases have been reported in calculations in one and two dimensional systems 13,14. These are structures of spins aligned in one direction with the sense varying periodically. For the values of $J_{\mathrm{AF}} / t$ considered here, we only find the $\left(\frac{\pi}{2}, \frac{\pi}{2}, \pi\right)$ (that corresponds $2 \times 2 \times 1$ blocks of equal spins $)$ and $\left(\frac{\pi}{3}, \pi, \pi\right)(3 \times 1 \times 1$ blocks $)$, although we have tried different combinations 15. In these phases, the electrons are localized, and the electronic density of states is built up of delta functions. Helix phases are ordinary spin density waves. It is interesting to note that helix phases are completely irrelevant in the thermodynamic limit, but they are remarkably stable on finite lattices, and should be considered when analyzing numerical simulations.

We find two new phases, intrinsic to the cubic lattice, labeled flux and skyrmion in Table I. The spin configuration is not coplanar, and the spins are parallel to the 
diagonals of the unit cube (note that the energy is invariant under a global rotation, so that only the relative angles are relevant). In the skyrmion phase, the spin directions around a unit cube point out from the center, with a hedgehog shape. The induced Berry phase can be thought of as generated by an effective magnetic monopole inside each of the unit cubes which build up the three dimensional lattice. The charge of the monopoles change sign in neighboring cubes, leading to zero total charge. The flux within each cube is isotropic, and equal to $\pi / 3$ (skyrmion configuration) and $2 \pi / 3$ (flux configuration) per plaquette. In both cases, the lattice unit contains eight sites, and the global symmetry remains cubic.

The electronic dispersion relation for all these phases can be calculated analytically, and it is given in Table I. The corresponding density of states is shown in Fig. 1. The dispersion relation in the flux and skyrmion phases depend linearly on $\boldsymbol{k}$ near $\boldsymbol{k}=\left( \pm \frac{\pi}{2}, \pm \frac{\pi}{2}, \pm \frac{\pi}{2}\right)$, and resembles the dispersion of Dirac massless particles in the lattice.

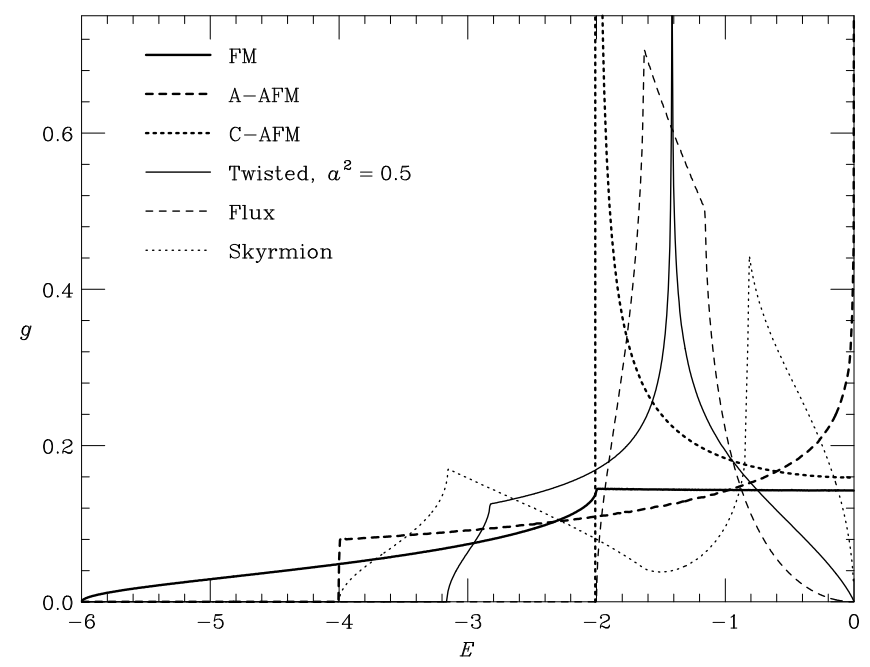

FIG. 1. Density of states for several spin configurations

The flux and twisted phases show similarities to the phase with spins at right angles found in two dimensions 29, 30, 14.

In the twisted phase, for $a=b=1 / \sqrt{2}$, the planes $x z$ and $y z$ have the same structure as the $2 \mathrm{D}$ pattern studied in 29, 30, 14. The flux phase, on the other hand, has a dispersion relation which is the natural extension to $3 \mathrm{D}$ of the one for the 2D flux phase. Note, however, that the flux phase in 2D is coplanar, and all phases in the hopping elements can be rendered real by an appropriate gauge transformation, while this does not happen in the 3D phase studied here.

The stability of the flux and skyrmion phases arise from the canting of the spin orientations, coupled to a shift in the electronic density of states toward the band edges. It is interesting to note that the electronic energy of the flux phase is close to that of the C-AFM one, and the energy of the skyrmion phase is also close to that of the A-AFM phase, for the entire range of electronic concentrations.

The phase diagram at zero temperature is shown in Fig. 2. All transitions are first order. The most interesting result is the stabilization of the skyrmion phase for a range of dopings and values of $J_{\mathrm{AF}} / t$ where the model is applicable to real materials. The twisted phase changes continuously from $a^{2}=0.45$ (right) to $a^{2}=0.77$ (left). Notice that although the A-AFM phase is beside the twisted phase, there is a discontinuity in the $a$ parameter $(a=0$ for A-AFM). We find island phases 14 in a more restricted range of parameters than in two dimensions, possibly due to the competition with the flux and skyrmion phases. We have not found any tridimensional island as $\left(\frac{\pi}{2}, \frac{\pi}{2}, \frac{\pi}{2}\right)$. For more negative values of $J_{\mathrm{AF}} / t$ we also find a $\left(\frac{\pi}{2}, \pi, \pi\right)$ island phase starting at -0.19 .

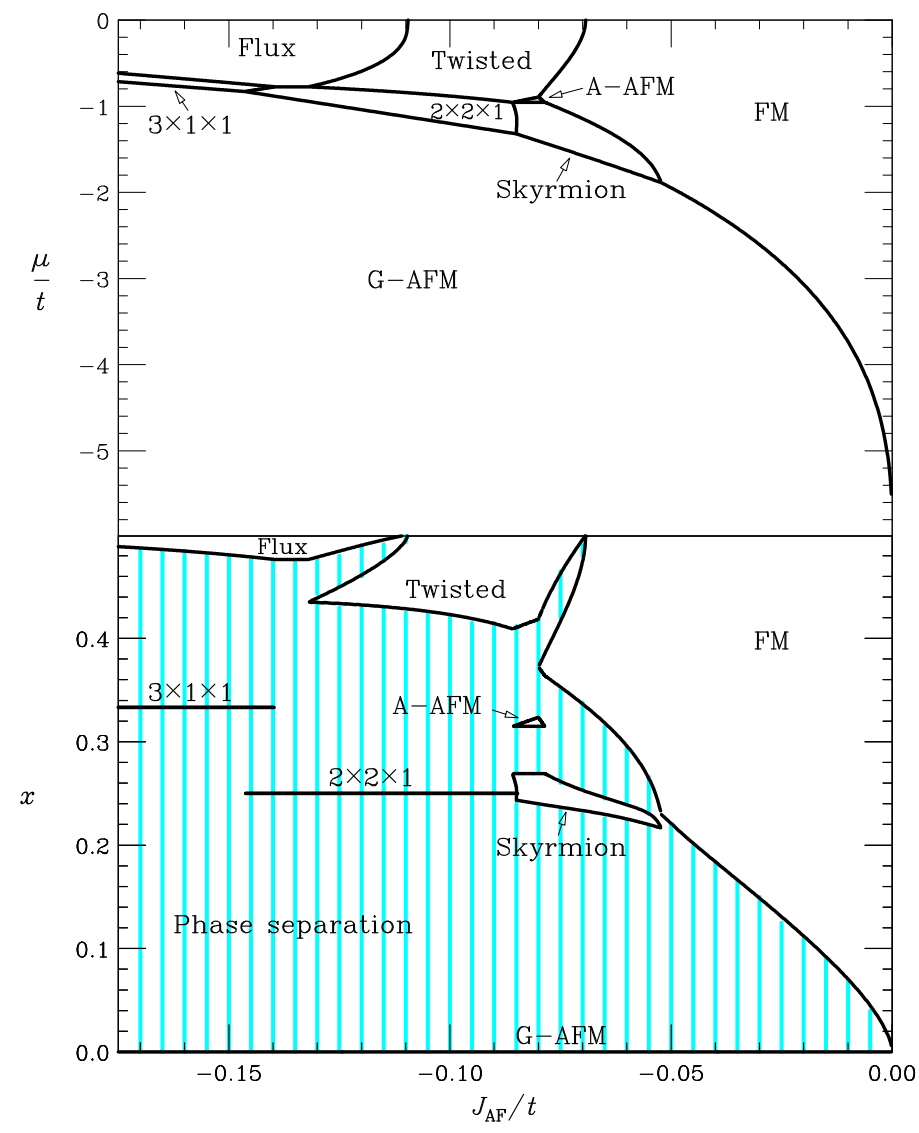

FIG. 2. Phase diagram at $T=0$. Upper part: chemical potential $(\mu / t)$ vs. $J_{\mathrm{AF}} / t$. Lower part: concentration $(x)$ vs. $J_{\mathrm{AF}} / t$. The results shown in this plot have been obtained in a $2400^{3}$ lattice. We have checked that the variations due to finite-size effects are smaller than the line widths. 


\begin{tabular}{lcc}
\hline \hline Type & spin direction & $\epsilon(\boldsymbol{k}) / t$ \\
\hline Ferromagnetic (FM) & $(0,0,1)$ & $-2 \sum_{\mu=1}^{3} \cos k_{\mu}$ \\
A-type $(\mathrm{A}-\mathrm{AFM})$ & $\left(0,0,(-1)^{z}\right)$ & $-2 \sum_{\mu=1}^{2} \cos k_{\mu}$ \\
C-type (C-AFM) & $\left(0,0,(-1)^{x+y}\right)$ & $-2 \cos k_{3}$ \\
G-type $(\mathrm{G}-\mathrm{AFM})$ & $\left(0,0,(-1)^{x+y+z}\right)$ & 0 \\
Twisted & $\left(a(-1)^{x+y}, b(-1)^{z}, 0\right)$ & $\pm 2 \sqrt{a^{2} \cos ^{2} k_{3}+b^{2}\left(\cos k_{1} \pm \cos k_{2}\right)^{2}}$ \\
Flux & $a^{2}+b^{2}=1$ & $\pm 2 \sqrt{\frac{1}{3} \sum_{\mu=1}^{3} \cos k_{\mu}}$ \\
Skyrmion & $\left((-1)^{y+z},(-1)^{x+z},(-1)^{x+y}\right) / \sqrt{3}$ & $\pm 2 \sqrt{\frac{2}{3}} \sqrt{\sum_{\mu=1}^{3} \cos ^{2} k_{\mu} \pm \sqrt{3 \sum_{\mu \neq \nu} \cos ^{2} k_{\mu} \cos ^{2} k_{\nu}}}$ \\
Helix & $\left((-1)^{x},(-1)^{y},(-1)^{z}\right) / \sqrt{3}$ & $-2 \sum_{\mu=1}^{2} \cos _{\mu}-2 \cos (\omega / 2) \cos \left(k_{3}+\omega / 2\right)$ \\
Island $\left(\frac{\pi}{2}, \pi, \pi\right)$ & $(\cos (\omega z), \sin (\omega z), 0)$ & $-1,1$ \\
Island $\left(\frac{\pi}{3}, \pi, \pi\right)$ & $\left(0,0,(-1)^{\left[\frac{x}{2}\right]+y+z}\right)$ & $-\sqrt{2}, 0, \sqrt{2}$ \\
Island $\left(\frac{\pi}{2}, \frac{\pi}{2}, \pi\right)$ & $\left(0,0,(-1)^{\left[\frac{x}{3}\right]+y+z}\right)$ & $-2,0,0,2$ \\
\hline \hline
\end{tabular}

Table I. Spin configurations and electronic dispersion relations of the different phases considered in the text. The notation [.] stands for the integer part. For a lattice of linear dimension $L, \omega$ and $k_{\mu}$ can be written as $\frac{2 \pi}{L} n$, with $n$ integer (in twisted, flux and skyrmion configurations $n$ is even as the unitary cell is a $2^{3}$ cube).

\section{B. Finite temperature results.}

We have extended the previous studies to finite temperatures by using the hybrid Monte Carlo method reported elsewhere [26], which allows to thermalize $16 \times 16 \times$ 16 clusters in reasonable computer time. We have studied the model (11), on the cubic lattice of side $L$ with periodic boundary conditions, using the grand-canonical ensemble (4). The use of an efficient algorithm has been crucial, because around two hundred points of the $\left(T, \mu, J_{\mathrm{AF}} / t\right)$ phase-diagram have been studied.

Before presenting our results it is worth to comment on the strong finite-size effects of this model. Fortunately, one can have a good analytical command on them (at least at zero temperature), by repeating the minimization of subsection III A on a finite lattice (this is straightforward using Table I). In this way one discovers that the phase boundaries change considerably with the lattice size. Even worse, for lattice sizes $L=4$ and 8, the helix phase of minimal frequency $\omega=2 \pi / L$ (see Table I) is more stable than the ferromagnetic phase in important regions of the phase diagram. Also the $\mathrm{C}-\mathrm{AFM}$ phase is stable in the $L=8$, although it is unstable in the $L \rightarrow \infty$ limit. On the other hand, the zero temperature phase di- agrams of the $L=6$ and $L=10$ lattices are rather close to the infinite volume limit. The only caveat of this lattice sizes is that they cannot accommodate island-phase whose unit cell is not commensurate with them (in particular, the $\left(\frac{\pi}{2}, \frac{\pi}{2}, \pi\right)$ island phase, that has a $4 \times 4 \times 2$ unit cell). We have therefore chosen to perform most of the numerical simulations on the $6^{3}$ cluster and, only when necessary for elucidation, we have employed as a larger cluster the $10^{3}$. Exception to this rule has been the simulation of the $8^{3}$ in the range $-0.29 \leq J_{\mathrm{AF}} / t \leq-0.18$ range, to check for the presence of the $2 \times 2 \times 1$ island phase.

In the simulation, we have used the perfect action formulation [26], with $\lambda=0.25$. The molecular dynamics parameters have been chosen to ensure an acceptance rate greater than an $80 \%$. The selection depends mainly on the temporal lattice size (inverse temperature). Typical values used are $N_{\tau}=20, \Delta \tau=0.02$ for $T / t=1 / 20$, and $N_{\tau}=50, \Delta \tau=0.01$ for $T / t=1 / 45$. We have systematically controlled the correct thermalization of our data, by comparing the results of hot and cold starts. In some cases, in particular at $T=t / 100$, we have also compared the results of ordered starts with a slow annealing from high temperatures. The total run-length 
(up to 2000 trajectories in some points) has always been at least larger than three times the thermalization time. The typical size of our statistical errors can be found in figure 3. The total CPU time devoted to this project has been the equivalent of 10 years of a Pentium III at 800 $\mathrm{MHz}$.

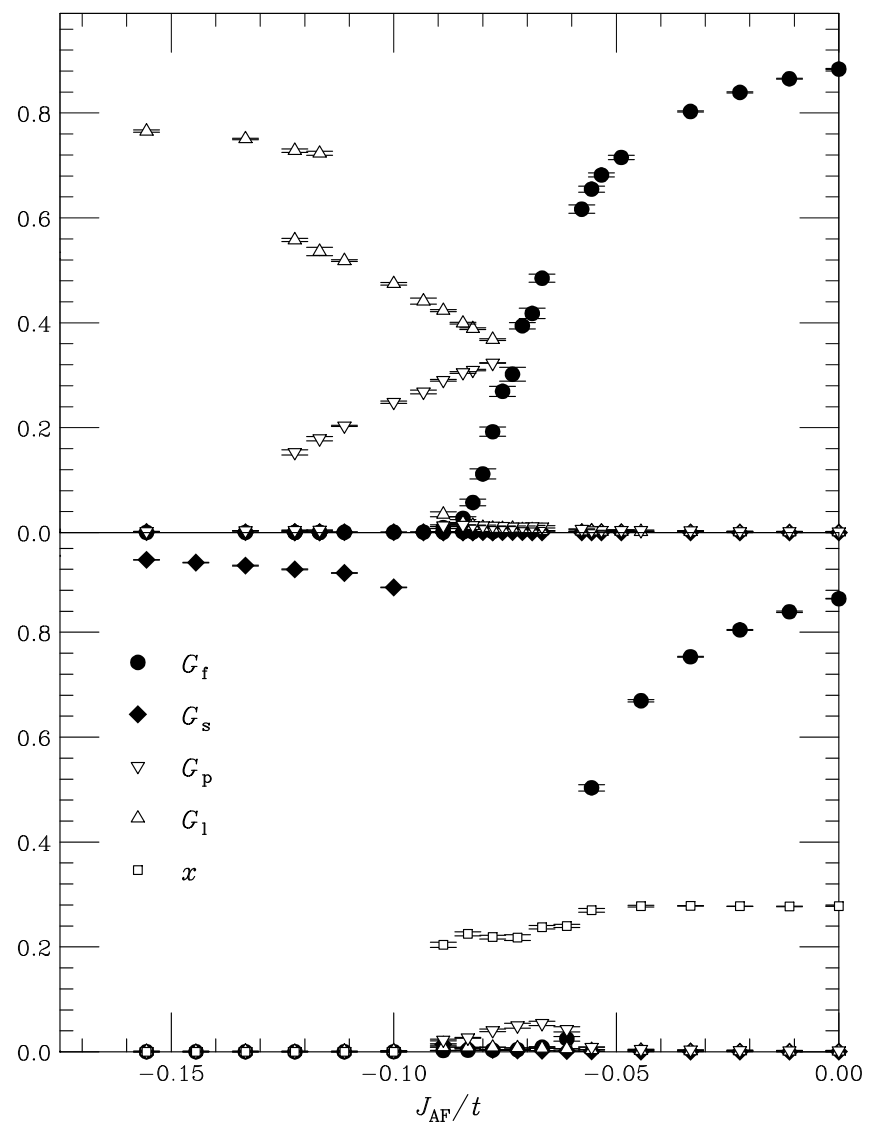

FIG. 3. Monte Carlo measures of several quantities at $T=t / 45$ for $\mu=0$ (upper side) and $\mu=-1.5$, on the $L=6$ lattice.

As we have said in the introduction, our scope has been the determination of the magnetic phase diagram of the model. Since one cannot break a symmetry on a finite lattice, it is necessary to use pseudo order-parameters for obtaining the phase diagram [32]. We have defined our pseudo order-parameters in terms of the spin structure factor ( $V=L^{3}$ is the total number of spins),

$$
\begin{aligned}
G(\boldsymbol{k}) & =\left\langle|\hat{\boldsymbol{S}}(\boldsymbol{k})|^{2}\right\rangle, \\
\hat{\boldsymbol{S}}(\boldsymbol{k}) & =\frac{1}{\sqrt{V}} \sum_{j} \boldsymbol{S}_{j} \mathrm{e}^{\mathrm{i} \boldsymbol{k} \cdot \boldsymbol{r}_{j}} .
\end{aligned}
$$

In the above equation, $\langle\cdot\rangle$ stands for the thermal average. In a paramagnetic phase, $G(\boldsymbol{k})$ will be of order $1 / V$ for all $\boldsymbol{k}$. On the other hand, on an ordered phase it will be of order one for a very small set of $\boldsymbol{k}$ 's (see below), and of order $1 / V$ for the rest of wave numbers. If one now calculates the structure factor for the configurations in Table I, it follows that the appropriate pseudo orderparameters are:

$$
\begin{aligned}
G_{\mathrm{f}} & =G(0,0,0), \\
G_{\mathrm{s}} & =G(\pi, \pi, \pi), \\
G_{\mathrm{l}} & =G(\pi, \pi, 0)+G(\pi, 0, \pi)+G(0, \pi, \pi), \\
G_{\mathrm{p}} & =G(\pi, 0,0)+G(0, \pi, 0)+G(0,0, \pi), \\
G_{\mathrm{h}} & =G\left(\frac{2 \pi}{L}, 0,0\right)+G\left(0, \frac{2 \pi}{L}, 0\right)+G\left(0,0, \frac{2 \pi}{L}\right) .
\end{aligned}
$$

Let us explain them in details. In a ferromagnetic phase, only $G_{\mathrm{f}}$ is of order 1, while for a G-AFM phase, the appropriate pseudo order-parameter would be $G_{\mathrm{s}}$. For an A-AFM phase, the selected order parameter will be $G_{\mathrm{p}}$ (we sum for $\boldsymbol{k}=(\pi, 0,0),(0, \pi, 0)$ and $(0,0, \pi)$ because we do not know a priori the direction of the AFM lattice planes). A skyrmion phase will also have $G_{\mathrm{p}}$ as pseudo order-parameter, and so the question arise of how to differentiate a skyrmion from an A-AFM phase. The easiest way to do that is to control that for the skyrmion phase the three quantities $G(\pi, 0,0), G(0, \pi, 0)$ and $G(0,0, \pi)$ are simultaneously large (we have also numerically checked the orthogonality of the Fourier transformed spin field at the three $\boldsymbol{k}$ values). Also the C-AFM phase and the flux phase have a common pseudo orderparameter, namely $G_{1}$, but one can differentiate both phases in exactly the same way. A twisted phase will have as pseudo order-parameter both $G_{\mathrm{p}}$ and $G_{\mathrm{l}}$. One can distinguish the twisted phase from a tunneling phenomena between an A-AFM and a C-AFM phases, checking again that both pseudo order-parameters are simultaneously large (we have also checked the orthogonality of the Fourier transformed spin-field at the two $\boldsymbol{k}$ for which the structure factor is not negligible). Finally the helix phase is signaled by the $G_{\mathrm{h}}$ order parameter, while the structure factor for the $\left(k_{1}, k_{2}, k_{3}\right)$ island phase is heavily peaked at $\boldsymbol{k}=\left(k_{1}, k_{2}, k_{3}\right)$.

The results for the $G_{\alpha}$ pseudo order-parameters and for the equilibrium fermionic density at $T / t=1 / 45$ are shown in Fig. 3, for $\mu=0$ (half filling, $x=0.5$ ) and $\mu=$ $-1.5 t$ which includes the interesting region of $x \approx 1 / 4$ $1 / 3$. The numerical results shown in this plot allow us to identify the FM, G-AFM, twisted and flux phases, and the onset of the skyrmionic phase.

For $\mu=0$, the flux-twisted transition is clearly discontinuous. In addition, there is a clear metastable behavior (the duplicated points correspond respectively to two metastable phases). At the right end of the twisted phase, $G_{\mathrm{p}}$ and $G_{\mathrm{l}}$ vanish abruptly and there are clear signs of metastability with a phase where $G_{\mathrm{f}}$ is small but non-vanishing. To check if it is a finite size effect, we have performed some simulations in this region with a larger lattice $\left(10^{3}\right)$, obtaining that $G_{\mathrm{f}}$ is much smaller (and $G_{\mathrm{p}}$ and $G_{\mathrm{l}}$ vanish). Thus we conclude that there is a first order transition at this temperature between the twisted and the paramagnetic phases. On the contrary, the ferromagnetic-paramagnetic transition is smoother. We recall that in Ref. [26] it is shown that at $J_{\mathrm{AF}}=0$, 
$T / t=0.14$ the transition is second order. Although we find a strengthening of this transition when $\left|J_{\mathrm{AF}}\right|$ grows, to confirm that it actually becomes of the first-order would require a careful finite-size scaling analysis that is beyond the scope of this work.

For $\mu=-1.5$ (see Fig. 3) we find a very different landscape. At the left part we find an almost saturated G-AFM region (notice that $x \approx 0$ and the fermions essentially play no role). In the center, we find a region with $x \approx 0.25$ where $G_{\mathrm{p}}$ is about 0.05 and the others are much smaller. We interpret this result as the onset of the skyrmionic region (that appears clearly at lower temperatures, see Fig. 5). The start of the ferromagnetic region is much abrupter than at $\mu=0$, but as said above, to conclude that it is first order, a study in different lattice sizes is mandatory.

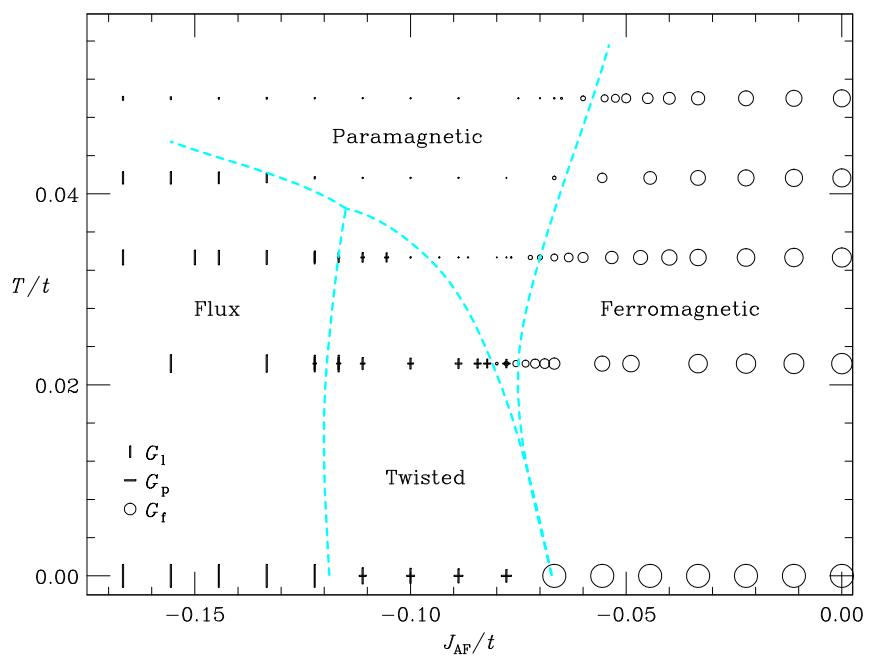

FIG. 4. Phase diagram obtained with Monte Carlo measures for $\mu=0$. The length of the segments, or the diameter of the circles are proportional to the associated $G_{\alpha}$. For clarity at each point we plot only the maximum $G_{\alpha}$ except in the cases where $G_{\mathrm{p}} / G_{1} \in[0.25,1]$, that correspond to the twisted phase.

To explore the phase diagram, we have simulated at several values of $T$. The phase diagram at half filling is plotted in Fig. 1. The paramagnetic region separates the ferromagnetic from the twisted phase for $T / t \geq 1 / 45$ but it is difficult to know the point where it finishes (maybe $T=0$ ). As the ferromagnetic magnetization goes to zero rather smoothly, to obtain a more precise value of the transition point we have used data from larger lattices (mainly $L=10$ ). The dashed lines have been draw approximately at the phase transitions.

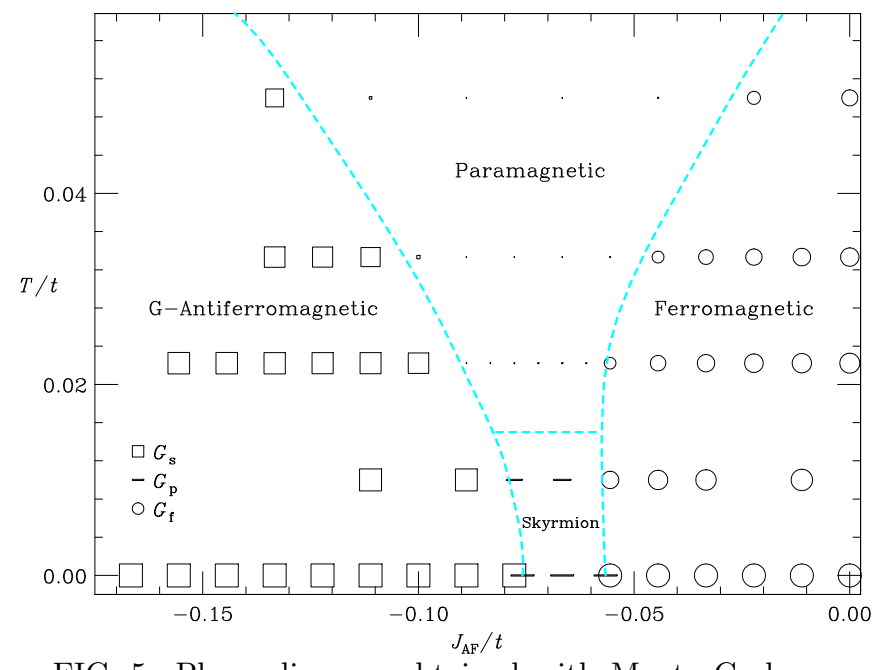

FIG. 5. Phase diagram obtained with Monte Carlo measures for $\mu=-1.5$.

Results for the different $G_{\alpha}$ order parameter for $\mu=$ $-1.5 t$ are shown in Fig. 5. Skyrmion correlations are suppressed at relatively low temperatures, but for $T=$ $t / 100$ we find a clear skyrmionic structure $\left(G_{\mathrm{p}}=0.7\right.$ - 0.8, with the appropriate orthogonality of the Fourier components).

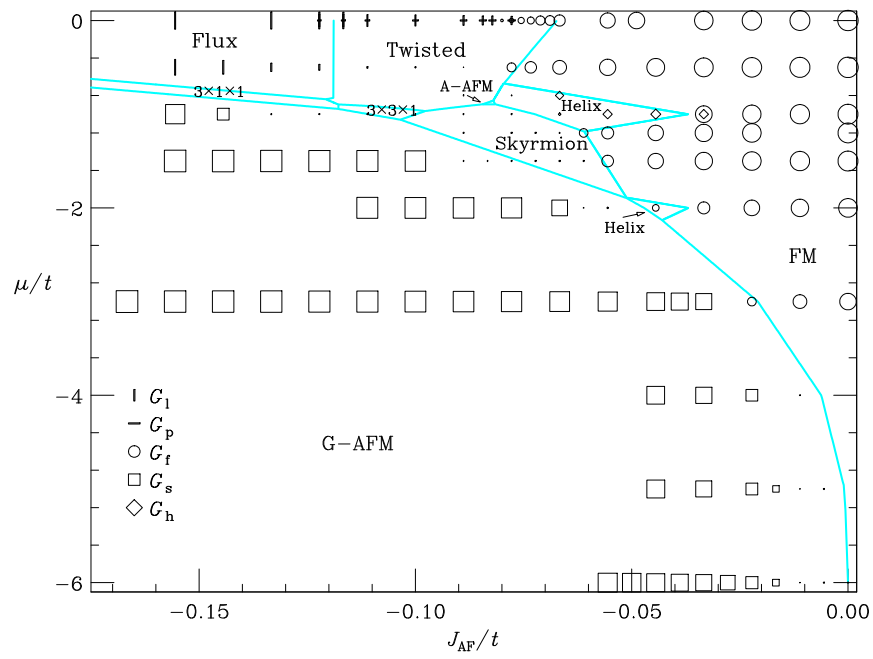

FIG. 6. Phase diagram at $T=t / 45$. The solid lines correspond to $T=0$ for a $L=6$ lattice.

We have combined all our results at $T=t / 45$ in the phase diagram shown in Fig. 6. The results are consistent with the zero temperature calculation for the $6^{3}$ lattice, whose transitions are plotted as continuous lines. The FM, G-AFM and Flux phases (and the twisted one near half filing) are less affected by finite temperature effects than the other phases, that become paramagnetic for temperatures as small as $T=t / 45$. Notice also that in the $6^{3}$ lattice the island phases are so narrow that they are hardly observed in a simulation. However, we have checked that in a $8^{3}$ lattice at $\mu=0, T=t / 45$ the system is in the $2 \times 1 \times 1$ phase for $J_{\mathrm{AF}} / t \in(-0.29,-0.18)$. 
We have also simulated a $8^{3}$ lattice in several values of $\left(\mu, J_{\mathrm{AF}}\right)$ that at $T=0$ correspond to the $2 \times 2 \times 1$ region, observing that even at $T=t / 45$ the system is disordered.

\section{The skyrmion phase.}

As mentioned above, we find a novel phase, the skyrmion phase, for a realistic range of dopings and antiferromagnetic couplings. We now discuss some physical features of this phase. Similar properties can be expected in the flux phase.

i) The symmetry of the magnetic phase is cubic. This phase is compatible with experiments done in the range $x \approx 1 / 4-1 / 3$ which show phases with cubic structure but no macroscopic magnetization 33.

ii) The magnetic arrangement is, at first sight, similar to that expected in a canted phase. Measurements of the microscopic magnetic structure will probably be indistinguishable from those expected for a canted phase.

iii) The magnon spectrum will resemble that of other systems with non collinear spins [34,35]. The dispersion relation will be linear at low energies.

iv) The phase is metallic. However, the chemical potential, at the appropriate dopings, coincides roughly with the position when the density of states has a pronounced minimum (see Fig. 1 ). Hence, it cannot be ruled out that residual disorder will induce insulating behavior. If this is the case, it will be behave in a similar way to the canted insulating phases reported in the literature (see, for instance [36]).

v) The unusual spin configuration leads to an effective coupling between an external magnetic field and a charge density wave. An applied field tilts the spins. The sign of the change in the angle between neighboring spins depends on the relative orientation of the spins and the magnetic field. A reduction in the angle leads to an increase of the hopping, and the opposite happens if the angle is increased. Hence, electron charge will be displaced from the weak bonds to the strong ones, and a charge density wave will arise. This feature can clearly identify the skyrmion phase.

\section{CONCLUSIONS.}

We have analyzed the phase diagram of the double exchange model in a cubic lattice, both at zero and at finite temperature. Our results at finite temperature have been obtained using an efficient Hybrid Monte Carlo algorithm [26], which has been crucial for the exploration of a three dimensional phase diagram. We have found that the competition between the ferromagnetic interaction mediated by the conduction electrons and antiferromagnetic couplings which arise from superexchange effects or from the underlying intra-atomic Hund's coupling lead to the existence of a variety of phases.
We have expressed our results in terms of the electron hopping, $t=W / 12$, where $W \sim 2 \mathrm{eV}$ is the width of the conduction band, and the effective antiferromagnetic coupling, $J_{\mathrm{AF}}$, arising from superexchange interactions between the $\mathrm{Mn} \mathbf{t}_{\mathbf{g}}$ orbitals $\left(\left|J_{\mathrm{AF}}\right| / t \sim 0.005-\right.$ 0.012 [37) and from the finite value of the intra-atomic Hund coupling, $J_{\mathrm{H}}$. It follows that the most phenomenologically interesting range for $\left|J_{\mathrm{AF}}\right| / t$ is $0.008-0.15$ [21]. Using the previous values, we find that the highest transition temperature $\left(x=0.5, J_{\mathrm{AF}}=0\right)$ is close to $350 \mathrm{~K}$, and therefore most of the phase transitions reported in this work occur in the $0-300 \mathrm{~K}$ range, in agreement with experiments.

It is somewhat surprising that conventional spin density wave structures are not stable. The three dimensional phase diagram shares some features with solutions of the double exchange model in one, two and infinite dimensions, like phase separation. A number of phases, however, have no counterparts in other dimensions.

We have identified, among others, a phase where the spins are locally arranged in a hedgehog manner, labelled skyrmion phase. The existence of this structure is probably intrinsic to the double exchange mechanism, and cannot be realized in spin systems with short range couplings. The skyrmion phase is stable for dopings $x \approx 0.2$ - 0.3 and antiferromagnetic couplings $\left|J_{\mathrm{AF}}\right| / t \approx 0.05$ 0.07 . These values are realistic for doped manganites in the range where Colossal Magnetoresistance effects are found. While it is probably difficult to characterize this phase experimentally, it will be interesting to verify its existence.

\section{ACKNOWLEDGEMENTS.}

We want to warmly thank Victor Laliena for a pleasant and fruitful collaboration. It is also a pleasure to thank B. Alascio, A. Aligia, E. Dagotto and B. Normand, for discussions on this problem.

We acknowledge financial support from grants PB960875, AEN97-1680, AEN97-1693, AEN99-0990 (MEC, Spain) and (07N/0045/98) (C. Madrid). V.M.-M. is supported by the Ministerio de Educación y Cultura. The simulations have been carried out in RTNN computers at Zaragoza and Madrid.

[1] E. D. Wollan and W. C. Koehler, Phys. Rev. 100, 545 (1955).

[2] D. I. Khomskii and G. Sawatzky, Solid State Commun. 102, 87 (1997).

[3] J. M. D. Coey, M. Viret and S. von Molnar, Adv. in Phys. 48, 167 (1999).

[4] C. Zener, Phys. Rev. 82, 403 (1951). 
[5] P. W. Anderson and H. Hasegawa, Phys. Rev. 100, 675 (1955).

[6] P.-G. de Gennes, Phys. Rev. 118, 141 (1960).

[7] E. Dagotto, T. Hotta and A. Moreo, preprint condmat/0012117).

[8] E. L. Nagaev, Physica B 230-232, 816 (1997).

[9] J. Riera, K. Hallberg and E. Dagotto, Phys. Rev. Lett. 79, 713 (1997).

[10] D. P. Arovas and F. Guinea, Phys. Rev. B 58, 9150 (1998)

[11] S. Yunoki, J Hu, A. L. Malvezzi, A. Moreo, N. Furukawa and E. Dagotto, Phys. Rev. Lett. 80, 845 (1998).

[12] D. Arovas, G. Gómez-Santos and F. Guinea, Phys. Rev. B 59, 13569 (1999).

[13] D. J. García, K. Hallberg, C. D. Batista, M. Avignon and B. Alascio, Phys. Rev. Lett. 85, 3720 (2000).

[14] H. Aliaga, B. Normand, K. Hallberg, M. Avignon and B. Alascio, preprint (cond-mat/0011342).

[15] We use the notation $\left(\frac{n_{1} \pi}{m_{1}}, \frac{n_{2} \pi}{m_{2}}, \frac{n_{3} \pi}{m_{3}}\right)$ that corresponds to the frequency associated to the maximum of the Fourier transform. Thus, $\left(\frac{\pi}{2}, \frac{\pi}{2}, \pi\right)$ and $\left(\frac{\pi}{3}, \pi, \pi\right)$ represent islands of sizes $2 \times 2 \times 1$ and $3 \times 1 \times 1$ respectively. We have studied all frequencies with the restrictions $m_{i} \leq 4, m_{1} m_{2} m_{3} \leq$ 9. In addition, we look at the phase $\left(\frac{\pi}{2}, \pi, \pi\right)+(\pi, \pi, \pi)$ (see Ref. 14] for the two dimensional case) that we find stable for $J_{\mathrm{AF}} / t$ values smaller than -0.3 far away from the region of physical interest.

[16] N. Furukawa, J. Phys. Soc. Jap. 64, 2734 (1995); ibid 64, 2754 (1995).

[17] F. Guinea, G. Gómez-Santos and D. P. Arovas, Phys. Rev.B 62, 391 (2000).

[18] K. Held and D. Vollhart, Phys. Rev. Lett. 84, 5168 (2000).

[19] A. Chattopadhyay, A. J. Millis and S. Das Sarma, Phys. Rev. B 61, 10738 (2000).

[20] N.-H. Tong and F.-C. Pu, Phys. Rev. B 62, 9425 (2000).

[21] J. L. Alonso, L. A. Fernández, F. Guinea, V. Laliena and V. Martín-Mayor, Phys. Rev. B 63, 64416 (2001).

[22] A. Moreo, M. Mayr, A. Feiguin, S. Yunoki, and E. Dagotto, Phys. Rev. Lett. 84, 5568(2000)

[23] S. Yunoki, T. Hotta, and E. Dagotto, Phys. Rev. Lett. 84, 3714 (2000).

[24] M. J. Calderón, L. Brey and J. A. Vergés, Phys. Rev. B 58, 3286 (1998).

[25] J. L. Alonso, L. A. Fernández, F. Guinea, V. Laliena and V. Martín-Mayor, Phys. Rev. B 63, 54411 (2001).

[26] J. L. Alonso, L. A. Fernández, F. Guinea, V. Laliena and V. Martín-Mayor, cond-mat/0007450, Nucl. Phys. B, in press.

[27] T. Mizokawa, D. I. Khomskii and G. A. Sawatzky, Phys. Rev.B 61, R3776 (2000); ibid 63, 024403 (2001).

[28] A. J. Millis, P. B. Littlewood, and B. I. Shraiman, Phys. Rev. Lett. 74, 5144 (1995).

[29] M. Yamanaka, W. Koshibae and S. Maekawa, Phys. Rev. Lett. 81, 5604 (1998).

[30] D. F. Agterberg and S. Yunoki, Phys. Rev. B 62, 13816 (2000).

[31] In small finite lattices, the stable island phases, with $4^{3}$ or $6^{3}$ unit cell appear only when compatible with the lattice size.
[32] In a finite lattice, the Monte Carlo average of a real order parameter like, for instance, the magnetization is zero also in a ferromagnetic phase. This is due to global rotations of the spins (global rotations cost no energy), that averages out the magnetization even if the instantaneous magnetization is large.

[33] J. M. De Teresa, C. Ritter, M. R. Ibarra, P. A. Algarabel, J. L. García-Muñoz, J. Blasco, J. García and C. Marquina, Phys. Rev. B 56, 3317 (1997).

[34] Th. Jolicoeur and J. C. Le Guillou, Phys. Rev. B 40, 2727 (1989).

[35] A. Mattsson, Phys. Rev. B 51, 11574 (1995).

[36] Y. Tomioka, A. Asamitsu, H. Kuwahara, Y. Moritomo and Y. Tokura, Phys. Rev.B 53, R1689 (1996).

[37] I. Panas, R. Glatt and T. Johnson, J. Phys. Chem. Solids 59, 2230 (1998). 